\title{
Highly efficient side-pumped solar laser with enhanced tracking-error compensation capacity
}

\author{
B. D. TibúrCiO ${ }^{1}$, D. LIANG $^{1 *}$, J. Almeida ${ }^{1}$, D. GARCIA ${ }^{1}$,C. R. VISTAS ${ }^{1}$ And P. J. Morais ${ }^{2}$ \\ ${ }^{1}$ CEFITEC, Departamento de Física, FCT, Universidade Nova de Lisboa, 2829-516 Campus de Caparica, Portugal \\ 2Instituto de Soldadura e Qualidade, Tagus Park-Oeiras, 2740-120 Porto Salvo, Portugal \\ *Corresponding author: dl@fct.unl.pt
}

\begin{abstract}
We report here a significant numerical improvement in side-pumped solar laser collection efficiency and solar-to-laser conversion efficiency, with an enhanced tracking error compensation capacity. Two side-pumped configurations, a single-rod and a dual-rod scheme were studied. The former pumped a thick laser rod with the full collection area of a parabolic mirror and the latter pumped two thin laser rods simultaneously, each rod being pumped by half of the collection area of the same concentrator. Both configurations were composed of a fused silica aspheric lens and a 2D-shaped-semicylindrical pump cavity, within which the Nd:YAG rods were mounted, allowing a tight focusing of the concentrated solar pump power from the focal spot of the heliostat-parabolic mirror solar energy collection and concentration system and an efficient pu33mping to the laser crystals. $42.70 \mathrm{~W}$ continuous-wave multimode solar laser power, corresponding to $27.37 \mathrm{~W} / \mathrm{m}^{2}$ collection efficiency, and 3.26\% solar-to-laser power conversion efficiency were numerically calculated for the single-rod scheme, being 1.55 and 1.34 times, respectively, higher than the previous state-of-the-art experimental records of side-pumped solar laser. For the dual-rod scheme, $37.72 \mathrm{~W}$ multimode solar laser power, corresponding to $24.18 \mathrm{~W} / \mathrm{m}^{2}$ collection efficiency, and $2.88 \%$ solarto-laser power conversion efficiency were numerically obtained, being 1.37 and 1.19 times, respectively, more than the previous record in side-pumping configurations. More importantly, largely enhanced brightness figure of merit, thermal performance and tracking error compensation capacity were attained by the dual-rod scheme, as compared to that of the single-rod scheme.
\end{abstract}

Keywords: Solar-pumped laser; multimode; dual-rod; single-rod; solar pumping; Nd:YAG

\section{Introduction}

Sun-powered lasers may be considered a future emerging technology for both renewable energy and laser technology, providing cost-effective solutions to laser radiation in an environmentally friendly way, especially in places where solar irradiance is abundant and other energy sources are scarce. Therefore, the direct production of narrow-band solar laser radiation by natural broad-band sunlight with high efficiency is of paramount importance for several laser applications such as space communications, atmospheric and ocean sensing or laser power beaming [1]. Solar pumped lasers have also great potential for terrestrial applications like high-temperature material processing and magnesium-hydrogen energy cycle [2, 3]. Nd:YAG solar laser continuous-wave (cw) emission at 1064 $\mathrm{nm}$, from $1.0 \mathrm{~W}$ in 1966 [4] to $60 \mathrm{~W}$ in 1988 [5], were successfully attained by Young and other researchers [5-9]. Solar laser collection efficiency(defined as solar laser power achieved per unit of area of the primary concentrator)was successfully increased to $6.7 \mathrm{~W} / \mathrm{m}^{2}$ in 2003 [8]. Significant technological advances have occurred after the application of Fresnel lenses as primary concentrators [9-12]. Collection efficiency of $30 \mathrm{~W} / \mathrm{m}^{2}$ was achieved in 2012 with a Nd:YAG rod pumped by a $4.0 \mathrm{~m}^{2}$ Fresnel lens and a liquid light guide lens [10]. Fresnel lenses have attractive advantages for solar laser research because of its reduced weight and cost, but with some practical inconveniences such as chromatic aberration, which is the dispersion of the full spectrum of the solar radiation along its focal zone [13]. In 2016, pumped by the NOVA heliostat-parabolic mirror collection and concentration system, $29.0 \mathrm{~W}$ cw solar laser power was registered, corresponding to $25 \mathrm{~W} / \mathrm{m}^{2}$ collection efficiency [14]. By using a medium 
size parabolic mirror at PROMES-CNRS (Procédés, Matériaux et Énergie Solaire-Centre National de la Recherche Scientifique), in France, $37.20 \mathrm{~W}$ cw laser output power was achieved in 2017 , corresponding to $31.50 \mathrm{~W} / \mathrm{m}^{2}$ collection efficiency [15]. The current record in solar laser collection efficiency is $32.50 \mathrm{~W} / \mathrm{m}^{2}$ by end-side-pumping a $4.5 \mathrm{~mm}$ diameter, $35 \mathrm{~mm}$ length $\mathrm{Cr}: \mathrm{Nd}: Y A G$ ceramic laser rod through NOVA heliostat-parabolic mirror system and a liquid guide lens as a secondary concentrator [16].

Although today's most efficient solar laser systems are all in end-side-pumping configurations [9-11, 15-17], sidepumping approach is also a very attractive choice for several reasons. The current record in solar laser collection efficiency is $17.60 \mathrm{~W} / \mathrm{m}^{2}$ by side-pumping a $3.0 \mathrm{~mm}$ diameter, $30 \mathrm{~mm}$ length $\mathrm{Nd}$ :YAG laser rod through NOVA heliostat-parabolic mirror system [18]. Side-pumping configuration is very adequate for laser power scaling and presents higher laser beam brightness as it allows a uniform absorption distribution along the rod axis, reducing the associated thermal induced effects. Besides, the free access to both rod ends permits the optimization of more laser resonator parameters, improving largely the laser beam quality and enabling the efficient extraction of the solar laser power.

Other advantage of side-pumped solar laser lies in its excellent solar tracking error compensation capacity as compared to that of the end-side-pumped configuration. In side-pumping configuration, solar tracking error in azimuth axis only influences the shift of focal spot along the laser rod, usually mounted in azimuth direction. The absorbed solar pump power within a pump cavity is therefore not considerably affected by the tracking errors in azimuth axis, as long as the focused spot still stays within the pump cavity. Also, since the variation of solar tracking error in altitude axis is not significant, especially at noon, we can usually achieve more stable solar laser output by side-pumping configuration. However, for end-side pumping an important part of the concentrated solar power at the focus is directly focused onto the end face of a laser rod, and any slight solar tracking error in either azimuth or altitude axis can cause a significant reduction or even extinction in laser output power, preventing its stable emission.

A large advance in side-pumped solar laser collection efficiency and solar-to-laser conversion efficiency, with enhanced tracking error compensation capacity is reported here. Two side-pumped configurations, a single-rod and a dual-rod pumping schemes were used. The former pumped a thick laser rod with the full collection area of the primary concentrator (commonly implemented in today's solar pumped lasers) and the latter pumped two thin laser rods simultaneously, each one using half of the collection area. For the single-rod scheme, $42.70 \mathrm{~W} \mathrm{cW}$ at 1064 $\mathrm{nm}$ solar laser power, corresponding to $27.37 \mathrm{~W} / \mathrm{m}^{2}$ collection efficiency and $3.26 \%$ solar-to-laser power conversion efficiency were numerically calculated, being 1.55 and 1.34 times, respectively, more than the previous experimental records in side-pumping configurations [18]. For the dual-rod scheme, $37.72 \mathrm{~W}$ solar laser power, corresponding to $24.20 \mathrm{~W} / \mathrm{m}^{2}$ collection efficiency, and $2.88 \%$ solar-to-laser power conversion efficiency were numerically obtained, being 1.34 and 1.19 times, respectively, more than the previous experimental records in sidepumping configurations [18]. More importantly, the dual-rod scheme presented 5.8 times enhancement in brightness figure of merit, a largely improved thermal performance, as compared to that of the single-rod scheme. The tracking error width at $10 \%$ laser power loss (TEW ${ }_{10 \%}$ ) was chosen to analyze the tracking error compensation capacity of both schemes. A large enhancement in tracking error compensation capacity was numerically achieved with the dual-rod pumping approach, where the $\mathrm{TEW}_{10 \%}$ was improved 1.66 and 1.21 times at altitude $(\Delta \mathrm{Y})$ and azimuth $(\Delta \mathrm{X})$ tracking errors, respectively, as compared to the numerically simulated side-pumped single-rod scheme with a thick rod. 


\section{NOVA heliostat-parabolic mirror solar system}

\subsection{NOVA heliostat-parabolic mirror solar energy collection and concentration system}

NOVA heliostat-parabolic solar system (Fig. 1) consisted of a large plane mirror, composed of 2 flat segments with $94 \%$ reflectivity, mounted on a two-axis heliostat, which redirected the incoming solar radiation towards a stationary parabolic mirror with $1.5 \mathrm{~m}$ diameter, $60^{\circ}$ rim angle and $660 \mathrm{~mm}$ focal length.

The parabolic mirror was back-surface silver coated and $80 \%$ reflectivity was assumed for this primary concentrator. The total combined reflectance of both the heliostat and the parabolic mirrors was $75 \%$. A shadowing area of 0.2 $\mathrm{m}^{2}$ was considered for the solar laser head, its supporting mechanics and the $X-Y-Z$ positioner, so $1.56 \mathrm{~m}^{2}$ effective solar collection area was considered.

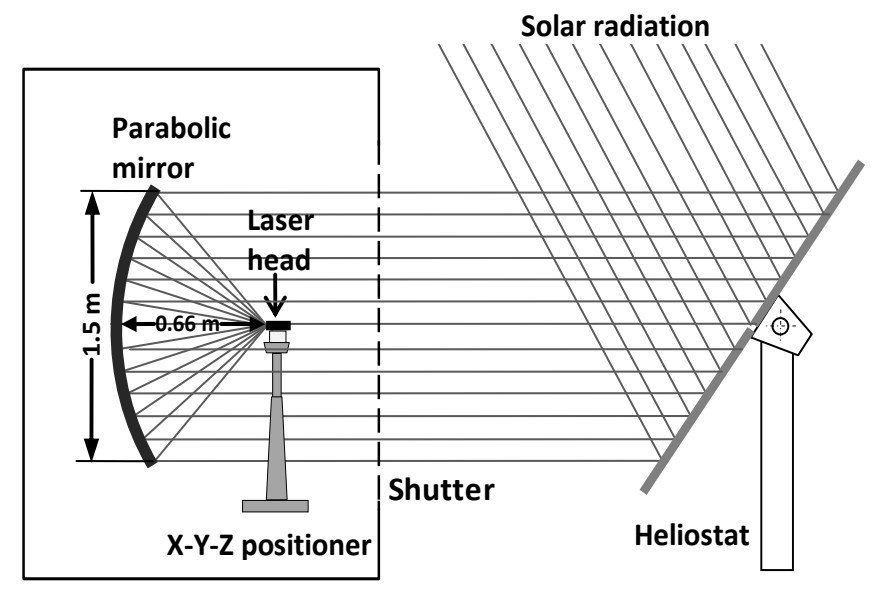

Fig. 1. Schematics of NOVA heliostat-parabolic mirror solar energy collection and concentration system.

With a typical solar irradiance of $890 \mathrm{~W} / \mathrm{m}^{2}$ in summer days in Lisbon, $1310 \mathrm{~W}$ can be measured at the focal spot of the parabolic mirror with near Gaussian distribution of $8.0 \mathrm{~mm}$ full width at half maximum (FWHM) in an optimal alignment condition of the heliostat.

\subsection{Nd:YAG solar laser head for both the single and the dual-rod pumping schemes}

As shown in Fig. 2, the solar laser head of the single-rod pumping scheme was composed of a fused silica aspherical lens and a 2D-shaped-semicylindrical pump cavity within which a $5.5 \mathrm{~mm}$ diameter and $25 \mathrm{~mm}$ length $\mathrm{Nd}$ :YAG rod was mounted and efficiently pumped. The laser rod was cooled by water in its longitudinal surface along $17 \mathrm{~mm}$ length and the remaining $8.0 \mathrm{~mm}$ was used for mechanical fixation by two rod holders. Both aspherical lens and the pump cavity were all actively cooled by water, which is also useful for partially preventing both UV solarization and IR heating to the laser rod. Fused silica is an ideal optical material for solar laser pumping because of its transparency over the Nd:YAG absorption spectrum, low coefficient of thermal expansion and resistance to thermal shock. The fused silica aspherical lens had an input face with $15 \mathrm{~mm}$ radius of curvature, $26 \mathrm{~mm}$ diameter, 0.002 conic factor and a plane output face, coupling efficiently the concentrated solar radiation from the focal zone to the input aperture of the pump cavity. The 2D-shaped pump cavity had $11 \mathrm{~mm} \times 14 \mathrm{~mm}$ rectangular input aperture and 8.0 $\mathrm{mm} \times 14 \mathrm{~mm}$ rectangular output aperture and a semicylindrical pump cavity with $5.0 \mathrm{~mm}$ radius, $14 \mathrm{~mm}$ length and $3.0 \mathrm{~mm}$ height. This pump cavity ensured one-pass, double-pass and even multi-pass absorption of the radiation by the laser material. $95 \%$ reflectivity was assumed for the inner wall of the reflectors of the pump cavity. 


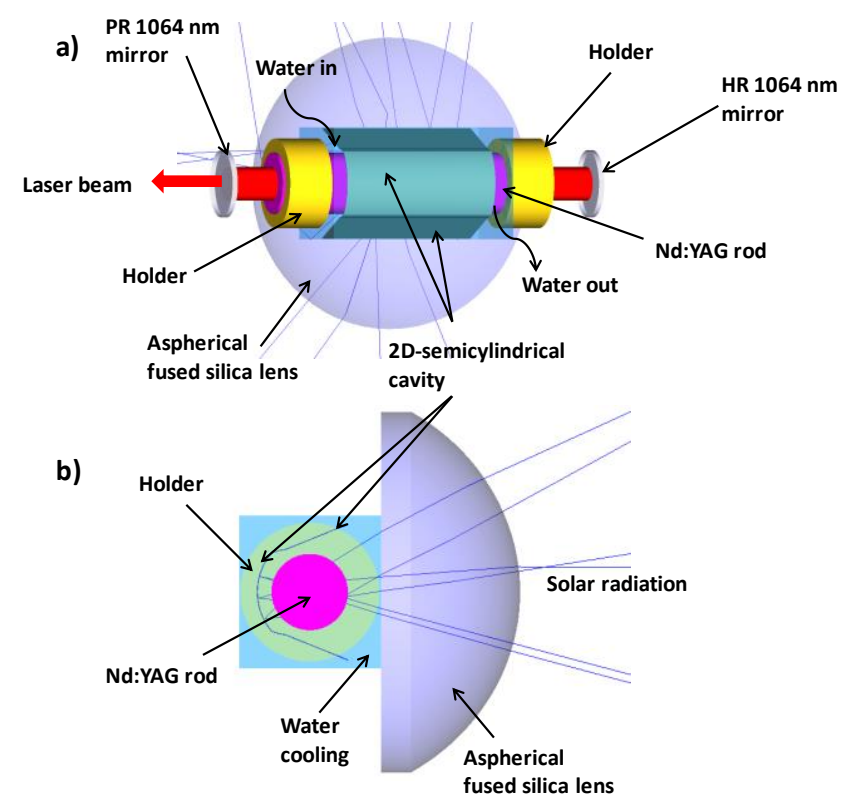

Fig. $2 \mathrm{Nd}$ :YAG rod solar laser head design in its traditional side-pumped single-rod scheme: a) 3-D view and b) front view. It is composed of the fused silica aspherical lens, the 2D-shaped-semicylindrical pump cavity and the Nd:YAG rod, fixed by two holders.

The laser cavity was formed by one high reflection (HR) $1064 \mathrm{~nm}$ mirror with 99.9\% reflectivity and another partial reflection (PR) $1064 \mathrm{~nm}$ mirror with $94 \%$ reflectivity (Fig. 2a).

For the dual-rod pumping scheme approach, as shown in Fig. 3, the fused silica aspherical lens had $15 \mathrm{~mm}$ radius of curvature, $26 \mathrm{~mm}$ diameter and 0.002 conic factor, coupling efficiently the concentrated solar radiation from the focal zone to the input aperture of the pump cavity, pumping the laser rod through the cooling water. The solar radiation was equally distributed from the output face of the fused silica lens into the apertures of the two 2D-shaped-semicylindrical cavities, mounted together with an angle of $33^{\circ}$ in relation to the optical axis. Each 2D-shaped pump cavity had $12 \mathrm{~mm} \times 14 \mathrm{~mm}$ rectangular input aperture and $11 \mathrm{~mm} \times 14 \mathrm{~mm}$ output aperture. Solar pump radiation emitted from the 2D-shaped cavity output ends could be efficiently coupled to the Nd:YAG rods through the semicylindrical pump cavities with $8.0 \mathrm{~mm}$ radius, $14 \mathrm{~mm}$ length and $3 \mathrm{~mm}$ height, producing one-pass, double-pass and even multi-pass absorption of the radiation by the laser material. 95\% reflectivity was assumed for the inner wall of the both elements of each pump cavity. The laser resonant cavity was formed by one HR $1064 \mathrm{~nm}$ mirror with 99.9\% reflectivity and another PR 1064 nm mirror with 95\% reflectivity. 


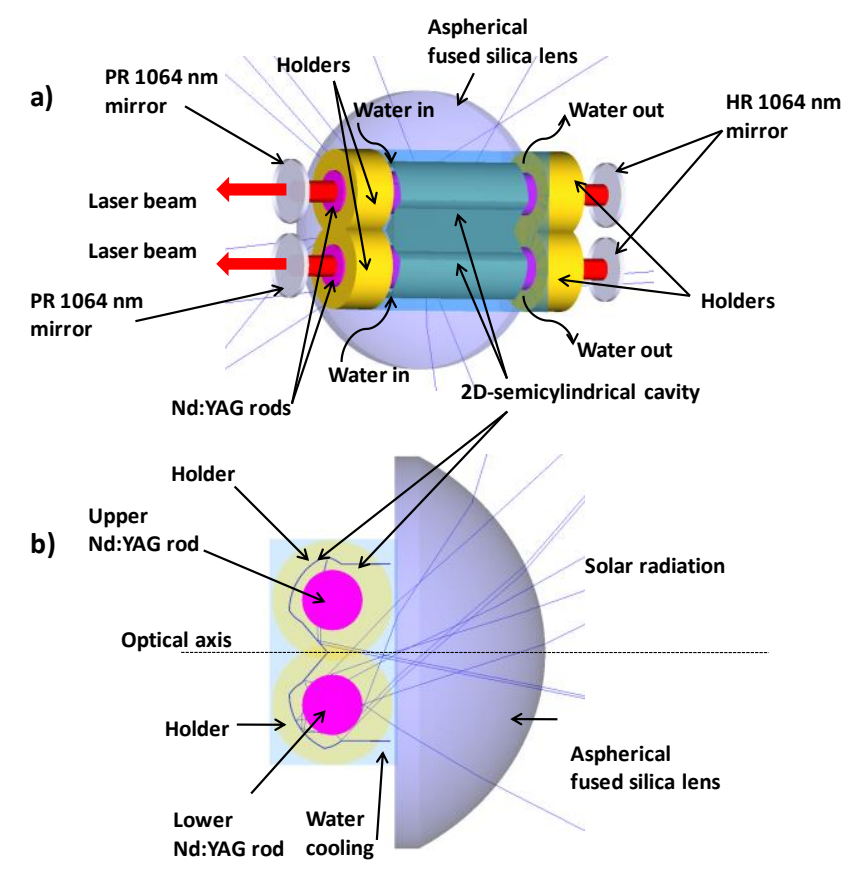

Fig. 3 Solar laser head design with two Nd:YAG laser rods: a) 3-D view and b) front view. It is composed of the fused silica aspherical lens, the two 2D-shaped-semicylindrical pump cavities and the two thick Nd:YAG rods, fixed by holders. The HR $1064 \mathrm{~nm}$ mirrors and the PR $1064 \mathrm{~nm}$ mirrors form the laser resonant cavities, along with the two laser rods.

\section{Numerical analysis of $\mathrm{Nd}$ :YAG solar laser performance for both the single and the dual-rod side-pumping schemes}

\subsection{ZEMAX ${ }^{\odot}$ non-sequential ray tracing analysis of both the single and the dual-rod side-pumping schemes}

Similar to our previous numerical analysis on solar lasers $[9,16,19]$, the Nd:YAG solar laser schemes above mentioned were optimized in its design parameters to achieve the maximum absorbed pump power, by nonsequential ray-tracing ZEMAX ${ }^{\odot}$ software. $890 \mathrm{~W} / \mathrm{m}^{2}$ solar irradiance was considered, as well as the half-angle of $0.27^{\circ}$ subtended by the Sun. The peak absorption of the useful wavelengths and their respective absorption coefficients of the laser medium were added to the glass catalog for 1.0 at.\% Nd:YAG laser material in ZEMAX ${ }^{\odot}$. The central wavelengths of these peaks were $527 \mathrm{~nm}, 531 \mathrm{~nm}, 568 \mathrm{~nm}, 578 \mathrm{~nm}, 586 \mathrm{~nm}, 592 \mathrm{~nm}, 732 \mathrm{~nm}, 736 \mathrm{~nm}$, $743 \mathrm{~nm}, 746 \mathrm{~nm}, 753 \mathrm{~nm}, 758 \mathrm{~nm}, 790 \mathrm{~nm}, 793 \mathrm{~nm}, 803 \mathrm{~nm}, 805 \mathrm{~nm}, 808 \mathrm{~nm}, 811 \mathrm{~nm}, 815 \mathrm{~nm}, 820 \mathrm{~nm}, 865 \mathrm{~nm}$, and $880 \mathrm{~nm}$. Spectral irradiance $\left(\mathrm{W} / \mathrm{m}^{2} / \mathrm{nm}\right)$ values corresponding to the above mentioned 22 peak absorption wavelengths could be consulted from the standard solar spectrum for one-and-a-half air mass (AM1.5) [20] and saved as source wavelength data in ZEMAX ${ }^{\odot}$ software. The absorption spectrum and the wavelength dependent refractive indexes of fused silica and water were also included in ZEMAX ${ }^{\odot}$ numerical data. The effective pump power of the light source took into account the $16 \%$ overlap between the Nd:YAG absorption spectrum and the solar spectrum [21]. The active laser medium was divided in 18000 zones in the ray-tracing analysis. The path length in each zone was found. With this value and the effective absorption coefficient, the absorbed power within the laser medium was calculated by summing up the absorbed pump radiation of all zones. 


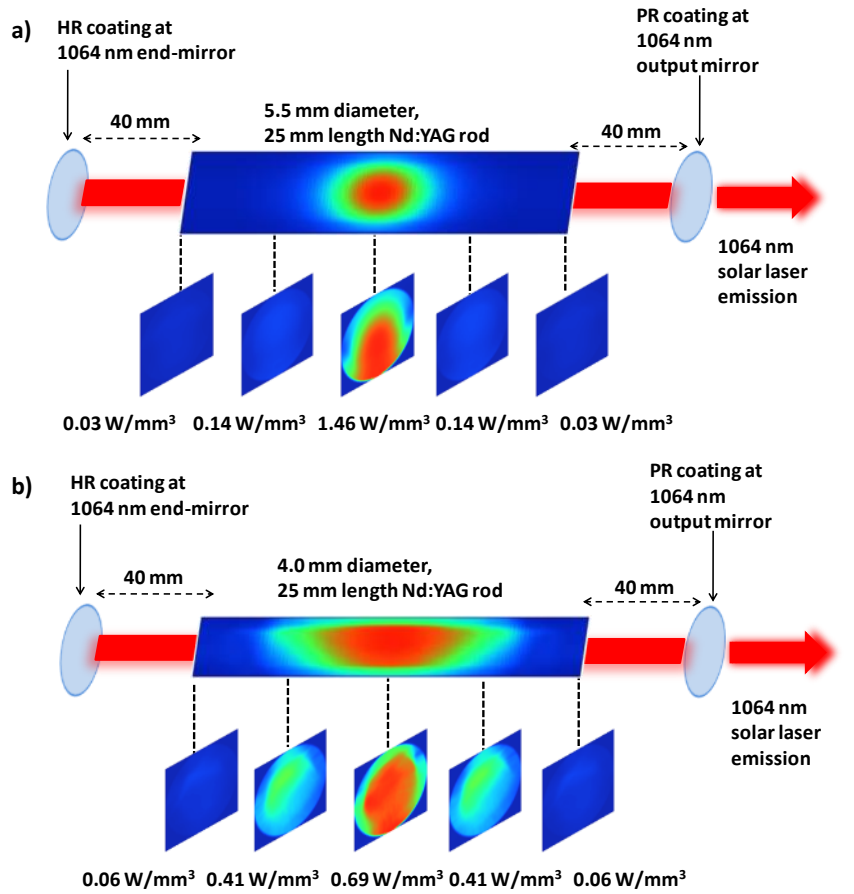

Fig. 4. Absorbed pump flux distributions along a) the $5.5 \mathrm{~mm}$ diameter, $25 \mathrm{~mm}$ length $\mathrm{Nd}: \mathrm{YAG}$ rod of the single-rod scheme and b) the $4.0 \mathrm{~mm}$ diameter, $25 \mathrm{~mm}$ length $\mathrm{Nd}$ :YAG rod of the dual-rod scheme.

The absorbed pump flux distributions along one longitudinal and five transversal central cross-sections of both the $5.5 \mathrm{~mm}$ diameter, $25 \mathrm{~mm}$ length Nd:YAG rod of the single-rod pumping scheme, and the $4.0 \mathrm{~mm}$ diameter, $25 \mathrm{~mm}$ length rod of the dual-rod pumping scheme, are shown in Fig. 4. The red color means maximum pump absorption while blue means little or no absorption.

The main contribution of the absorbed pump power comes from the center of the laser rod, especially in the case of the single-rod pumping scheme. In the case of the proposed dual-rod pumping scheme, a reduced peak intensity of about $52 \%$ was found in relation to that of the single-rod scheme. The absorbed pump flux data from ZEMAX ${ }^{\odot}$ software was then exported and processed by LASCAD $^{\odot}$ software for optimization of the laser resonator parameters.

3.2. LASCAD laser resonant cavity analysis of the multimode solar laser output performances for both the single and the dual-rod pumping schemes

Laser resonant cavity analysis and optimization of the laser output power were carried out using LASCAD ${ }^{\odot}$ software. The effective pump power of the light source took into account about $16 \%$ overlap between the absorption spectrum of the 1.0 at\% Nd:YAG laser medium and the solar spectrum [21]. Nd:YAG crystal offers advantages over other laser materials, despite its small value of overlap, such as good thermal conductivity $\left(\mathrm{K}=14 \mathrm{~W} \mathrm{~m}^{-1} \mathrm{~K}^{-1}[22]\right)$, high quantum efficiency and tensile strength $\left(\sigma=200 \mathrm{~N} / \mathrm{mm}^{2}\right.$ [22]). The stimulated emission cross-section of $2.8 \times 10^{-19} \mathrm{~cm}^{2}$, a fluorescence life time of $230 \mu \mathrm{s}$ [22], and an absorption and scattering loss of 0.003 $\mathrm{cm}^{-1}$ for the 1.0 at\% Nd:YAG medium were adopted in the LASCAD ${ }^{\odot}$ analysis. The mean absorbed and intensityweighted solar pump wavelength of $660 \mathrm{~nm}$ [5] was also assumed in the calculations. $0.2 \%$ and $0.1 \%$ imperfect optical coating losses were assumed for the laser rods and the HR1064nm end mirror, respectively. The diffraction losses depend strongly on rod diameter, resonator length and radius of curvature (RoC) of the resonator. LASCAD ${ }^{\odot}$ beam propagation method predicted $0.02 \%$ and $0.03 \%$ diffraction losses for the $5.5 \mathrm{~mm}$ diameter single-rod and for the $4.0 \mathrm{~mm}$ diameter dual-rod schemes, respectively. 


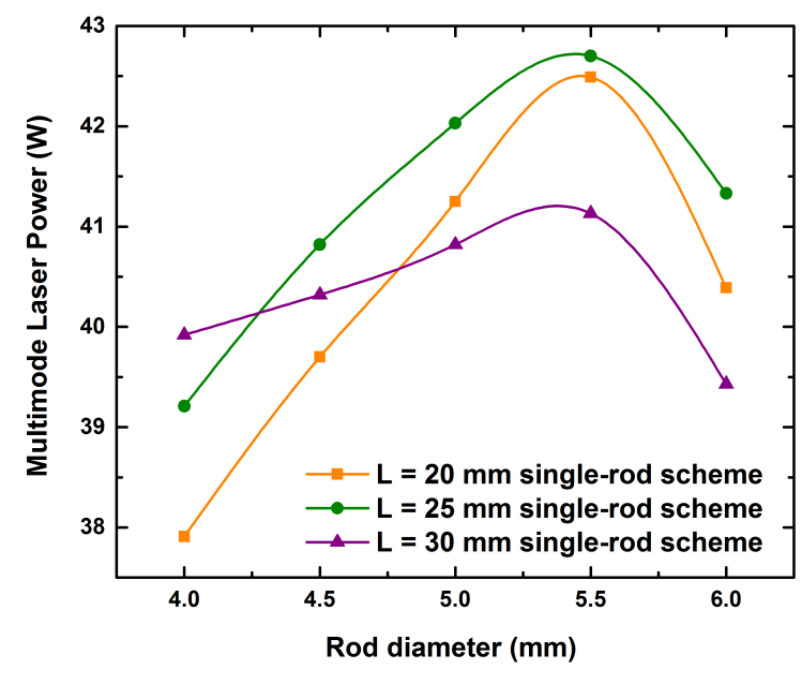

Fig. 5. Numerically calculated multimode laser output power as a function of both rod diameter and length for the single-rod scheme.

The laser output power in both schemes was numerically maximized using mirrors of different reflectivities and laser rods of different diameters and lengths. Through the adoption of a symmetrical laser resonant cavity, the multimode solar laser output power was efficiently extracted. Different RoC and resonant cavity lengths were tested for several rod diameters and lengths. The maximized numerical multimode laser output power for the single-rod scheme was achieved with a $105 \mathrm{~mm}$ length resonant cavity, and $\mathrm{RoC}=-10 \mathrm{~m}$ for both end-mirrors (Fig. 4). For the dual-rod scheme, the same parameters were found for achieving the maximum multimode laser output power.

The multimode laser output power for the single-rod scheme as a function of the laser rod diameter and length is shown in Fig. 5 . With $5.5 \mathrm{~mm}$ diameter and $25 \mathrm{~mm}$ rod length, the maximum multimode laser output power of 42.70 W was obtained.

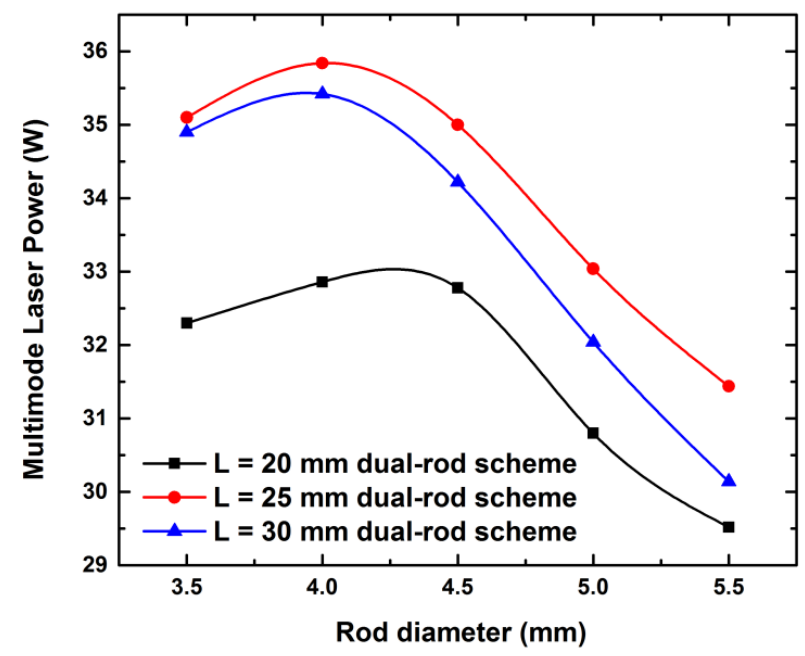

Fig. 6. Numerically calculated multimode laser output power as a function of both rod diameter and length for the dual-rod scheme with combined laser power.

The multimode laser output power as a function of the rod diameter and length for the dual-rod scheme is shown in Fig. 6. 
The total maximum laser output power of $35.84 \mathrm{~W}$ was obtained from the combined laser output power of $17.63 \mathrm{~W}$ from each of the $4.0 \mathrm{~mm}$ diameter and $25 \mathrm{~mm}$ length rods.

The possibility of extracting one single laser beam from the two rods can constitute an interesting solution to improve the $\mathrm{M}^{2}$ factors and consequently the brightness figure of merit of future solar-pumped lasers. Using a U-shaped resonant cavity, such as in diode-pumped rod lasers, through a single folded laser cavity [23], one laser beam can be obtained from the two laser rods, as shown in Fig. 7, using RoC $=-10 \mathrm{~m}$. The laser resonant cavity was formed by one HR $1064 \mathrm{~nm}$ end mirror with 99.9\% reflectivity (0.1\% loss), two HR $1064 \mathrm{~nm}$ folding mirrors with 99.9\% reflectivity (0.1\% loss each) and one PR $1064 \mathrm{~nm}$ output mirror with $95 \%$ reflectivity. $0.4 \%$ total imperfect optical coating losses for the two laser rods were assumed. LASCAD ${ }^{\odot}$ beam propagation method predicted $0.03 \%$ diffraction losses for the $4.0 \mathrm{~mm}$ diameter dual-rod scheme, resulting in the total round-trip loss of $3.73 \%$, as explained in section 3.3 .

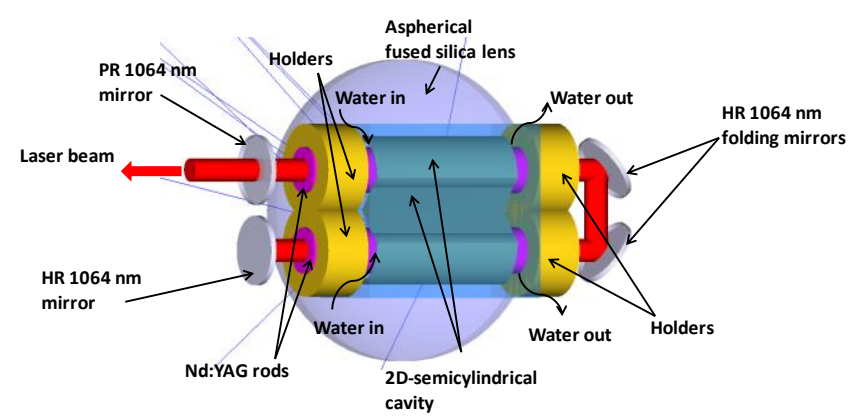

Fig. 7. Proposed design of the solar laser head with two Nd:YAG rods to obtain one single laser beam from the two rods. Two HR $1064 \mathrm{~nm}$ folding mirrors, one HR 1064 nm mirror and one PR 1064 nm mirror compose the U-shaped laser resonant cavity, along with the two laser rods.

The multimode laser output power as a function of the incoming solar power reaching the input face of the primary solar concentrator was also numerically calculated for all the schemes, as shown in Fig. 8. The laser power of each individual rod (both upper and lower rod), as well as its combined laser power, is also represented.

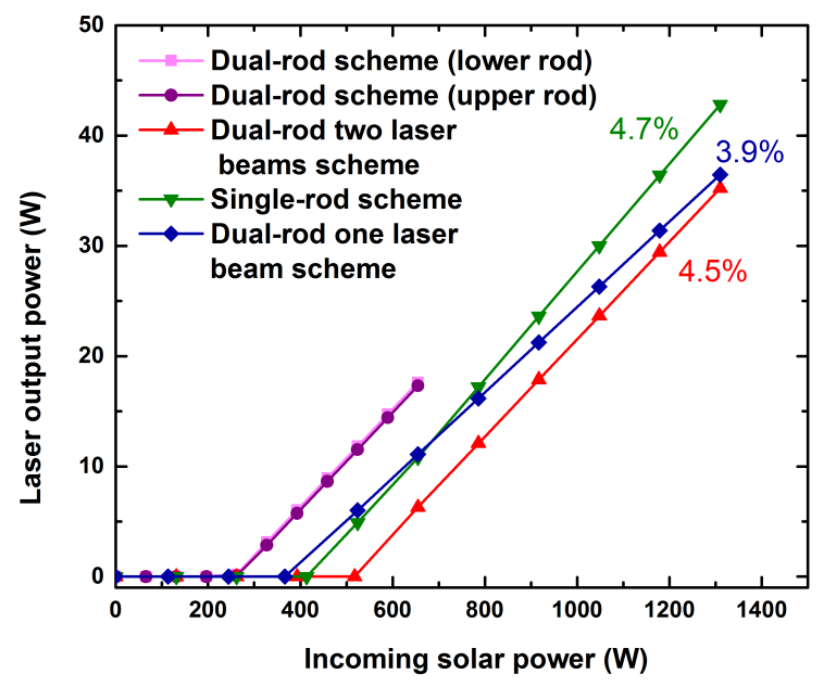

Fig. 8. Numerically calculated multimode laser output power as a function of the incoming solar power for both the single-rod and the dual-rod schemes. The latter in two configurations, two laser beams and one laser beam schemes. The solar laser slope efficiencies are also represented. 
The multimode laser slope efficiency - the slope of the curve obtained by plotting the laser output versus the incoming solar power - the $\mathrm{M}^{2}$ factors and the brightness figure of merit [8] were also numerically calculated for all the schemes. A threshold pump power of $413 \mathrm{~W}$ was numerically calculated for the single-rod scheme. $258.5 \mathrm{~W}$ threshold pump power was calculated for either the upper or the lower rod, resulting in the combined threshold pump power of $517 \mathrm{~W}$ for the dual-rod two laser beams scheme. However, for the dual-rod one laser beam scheme, the threshold pump power was reduced to $366 \mathrm{~W}$. The single-rod scheme offered the highest laser output power and laser slope efficiency, but the lowest brightness figure of merit. The dual-rod one laser beam scheme presented the highest brightness figure of merit amongst the studied schemes. The numerical performance of the single-rod, the dual-rod two laser beams and the dual-rod one laser beam schemes are summarized in Table 1.

Table 1. Numerically calculated multimode laser output power, collection efficiency, solar-to-laser power conversion efficiency, slope efficiency, threshold pump power, $\mathrm{M}^{2}$ factors and brightness figure of merit for the single-rod scheme, the dual-rod two laser beams and the dual-rod one laser beam schemes.

\begin{tabular}{|c|c|c|c|}
\hline & \multicolumn{3}{|c|}{ Schemes } \\
\hline Parameter & Single-rod & $\begin{array}{l}\text { Dual-rod two } \\
\text { laser beams }\end{array}$ & $\begin{array}{l}\text { Dual-rod one } \\
\text { laser beam }\end{array}$ \\
\hline Laser output power (W) & 42.70 & 35.84 & 37.72 \\
\hline Collection efficiency $\left(\mathrm{W} / \mathrm{m}^{2}\right)$ & 27.37 & 22.97 & 24.18 \\
\hline $\begin{array}{c}\text { Solar-to-laser power } \\
\text { conversion efficiency (\%) }\end{array}$ & 3.26 & 2.74 & 2.88 \\
\hline Slope efficiency (\%) & 4.70 & 4.50 & 3.90 \\
\hline $\begin{array}{c}\text { Threshold } \\
\text { pump power }(\mathrm{W})\end{array}$ & 413 & 517 & 366 \\
\hline$M^{2}$ factor $\left(M^{2} x ; M^{2} y\right)$ & $95.0 ; 95.0$ & $39.0 ; 39.0$ & $36.3 ; 36.3$ \\
\hline $\begin{array}{l}\text { Brightness figure of merit } \\
\text { (W) }\end{array}$ & 0.005 & 0.024 & 0.029 \\
\hline
\end{tabular}

\subsection{Analytical calculation of solar laser output power, slope efficiency and threshold pump power}

The laser output power $\left(P_{\text {out }}\right)$ can be analytically calculated in terms of incoming solar power $\left(P_{\text {inc }}\right)$ and other quantities by Eq. (1) [22]:

$$
P_{\text {out }}=\left(\frac{1-R}{1+R}\right)\left(\frac{2 \eta_{\text {OVP }} \eta_{T} \eta_{A} \eta_{Q} \eta_{S} \eta_{B}}{\delta-\ln R} P_{\text {inc }}-A l_{S}\right)
$$

Where $I_{S}$ is the saturation intensity, $A$ the cross-section area of the laser rod and $R$ the reflectivity of the output coupler. The optical losses in the resonator can be combined with the losses in the crystal through $\delta=21 \alpha+\delta_{M}+\delta_{D}$. The factor $2 / \alpha$ gives the two way loss inside the resonator, where $\alpha$ is the absorption and scattering coefficient of the laser material and/the length of the laser medium. $\delta_{M}$ is the miscellaneous losses related to the imperfect optical coating losses of both laser rod end faces and HR1064nm mirrors. $\delta_{D}$ the diffraction losses of the resonant cavity [22]. $\eta_{\text {ovp }}$ represents the overlap between the absorption spectrum of the laser medium and the solar emission spectrum, which is 0.16 for the Nd:YAG medium [21]. $\eta_{T}$ gives the fraction of the useful pump radiation, which is transferred from the entrance of the primary concentrator to the laser medium. From $\eta_{A}$ comes the fraction of the 
transferred useful radiation absorbed by the laser material. Using ZEMAX ${ }^{\odot}$ analysis, $\eta_{T}=0.78$ and $\eta_{A}=0.87$ were obtained for the single-rod scheme and $\eta_{T}=0.74$ and $\eta_{A}=0.82$ for the dual-rod scheme. $\eta_{Q}$ and $\eta_{S}$ are the quantum efficiency and Stokes factor, respectively. For the mean absorbed and intensity weighted pump wavelength of $660 \mathrm{~nm}, \eta_{S}=0.62$ was obtained [5]. $\eta_{B}$ is the beam overlap efficiency, which is the spatial overlap between the resonator modes and the absorbed pump distribution within the laser medium. The value of 0.91 was numerically attained for $\eta_{B}$ in LASCAD $^{\odot}$ analysis. The other quantities used in Eq. (1) are summarized in Table 2.

Table 2. Parameters used in solar laser slope efficiency, threshold pump power and laser output power analytical calculations.

\begin{tabular}{c|cc}
\hline Parameter & Single-rod scheme & Dual-rod scheme \\
\hline$A$ & $0.24 \mathrm{~cm}^{2}$ & $0.25 \mathrm{~cm}^{2}$ \\
$\alpha$ & $2.5 \mathrm{~cm}$ & $2.5 \mathrm{~cm}$ \\
$\delta_{M}$ & $0.003 \mathrm{~cm}^{-1}$ & $0.003 \mathrm{~cm}^{-1}$ \\
$\delta_{D}$ & 0.003 & 0.003 \\
$I_{S}$ & 0.0002 & $(0.007$ with folding mirrors) \\
$\eta_{T}$ & $2.9 \mathrm{~kW} / \mathrm{cm}^{2}[22]$ & 0.0003 \\
$\eta_{A}$ & 0.78 & $(0.0004$ with folding mirrors) \\
$\eta_{\text {OVP }}$ & 0.87 & $2.9 \mathrm{~kW} / \mathrm{cm}^{2}[22]$ \\
$\eta_{Q}$ & $0.16[21]$ & 0.74 \\
$\eta_{S}$ & $0.90[22]$ & 0.82 \\
$\eta_{B}$ & $0.62[5]$ & $0.16[21]$ \\
& 0.91 & $0.90[22]$ \\
& & $0.62[5]$ \\
\hline
\end{tabular}

Through Eq. (2) [22], the slope efficiency, $\eta_{\text {slope, }}$ is given by:

$$
\eta_{\text {slope }}=\left(\frac{1-R}{1+R}\right)\left(\frac{2 \eta_{O V P} \eta_{T} \eta_{A} \eta_{Q} \eta_{S} \eta_{B}}{\delta-\ln R}\right)
$$

Through Eq. (3) [22], the threshold pump power, $P_{\text {th }}$, is given by:

$$
P_{t h}=\frac{\delta-\ln R}{2 \eta_{O V P} \eta_{T} \eta_{A} \eta_{Q} \eta_{S} \eta_{B}} A I_{S}
$$

For the dual-rod one laser beam scheme with folding mirrors, $\delta_{M}=0.007$ and $\delta_{D}=0.0004$ were assumed. The numerical and analytical calculations of the laser output power, slope efficiency and threshold pump power for the single-rod scheme and the dual-rod scheme with two laser beams and one laser beam are summarized in Table 3. 
Table 3. Numerically and analytically calculated laser output power, slope efficiency and threshold pump power, for the single-rod scheme and the dual-rod scheme with two laser beams and one laser beam.

\begin{tabular}{cccc}
\hline & \multicolumn{3}{c}{ Parameters } \\
\hline & $\begin{array}{c}\text { Laser output power } \\
(\mathrm{W})\end{array}$ & $\begin{array}{c}\text { Slope efficiency } \\
(\%)\end{array}$ & $\begin{array}{c}\text { Threshold pump } \\
\text { power (W) }\end{array}$ \\
\hline Analytical calculation & & & \\
\hline Single-rod & 34.70 & 4.00 & 445 \\
Dual-rod two laser beams & 28.50 & 3.67 & 535 \\
Dual-rod one laser beam & 30.81 & 3.31 & 381 \\
\hline Numerical calculations & & & \\
\hline Single-rod & 42.70 & 4.70 & 413 \\
Dual-rod two laser beams & 35.84 & 4.50 & 317 \\
Dual-rod one laser beam & 37.72 & 3.90 & 366 \\
\hline
\end{tabular}

The analytical equations present only approximated values due to the fact that do not rely upon the detailed absorbed pump distribution within the laser rod. Through ZEMAX ${ }^{\odot}$ and LASCAD ${ }^{\odot}$ software more accurate values can be calculated since they count upon a detailed three dimensional absorbed pump power distribution and its thermal effects within the laser crystal.

\subsection{Improvement in Nd:YAG solar laser thermal performance}

A numerical analysis was carried out using $\operatorname{LASCAD}^{\odot}$ software in order to investigate the thermal conditions of the $\mathrm{Nd}: Y A G$ rods. Integrating the absorbed pump flux data of ZEMAX ${ }^{\odot}$ software over the whole laser medium volume, the heat load, the temperature and the stress intensity with the solar pumping radiation were analyzed for both the $4.5 \mathrm{~mm}$ diameter, $25 \mathrm{~mm}$ length Nd:YAG rod in the single-rod scheme and the $4.0 \mathrm{~mm}$ diameter, 25 length Nd:YAG rod in the dual-rod scheme. The results are shown in Fig. 9, as well as the reduction in prejudicial thermal induced effects.

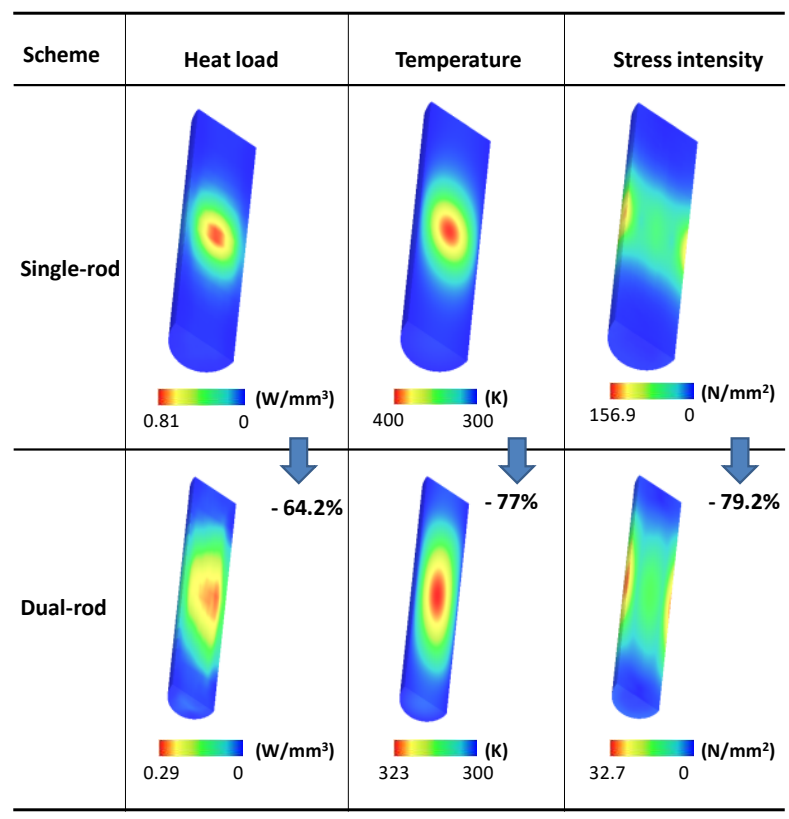

Fig. 9. Numerically calculated heat load, temperature and stress intensity distributions of the $5.5 \mathrm{~mm}$ diameter $25 \mathrm{~mm}$ length Nd:YAG rod from the single-rod scheme, and the $4.0 \mathrm{~mm}$ diameter $25 \mathrm{~mm}$ length Nd:YAG rod from the dual-rod scheme. 
In the case of the single-rod scheme, $0.43 \mathrm{~W} / \mathrm{mm}^{3}$ heat load, $344 \mathrm{~K}$ temperature and $64.30 \mathrm{~N} / \mathrm{mm}^{2}$ stress intensity were obtained. For the dual-rod scheme a maximum heat load of only $0.33 \mathrm{~W} / \mathrm{mm}^{3}$ was calculated. The temperature reduced to $321 \mathrm{~K}$ and a reduced stress intensity of $28.20 \mathrm{~N} / \mathrm{mm}^{2}$ was numerically attained. Comparing the thermal conditions, the thermal performance of the dual-rod scheme was significantly improved in relation to the single-rod scheme.

\section{Tracking error compensation capacity analysis of the dual-rod and the single-rod schemes}

The single and the dual-rod schemes were studied regarding to the tracking error capacity. Both schemes were pumped by NOVA heliostat-parabolic solar collection and concentration system with the same collection area of $1.56 \mathrm{~m}^{2}$. In Fig 10 is shown an example of the influence of the solar tracking error on the dual-rod scheme in altitude $(\Delta \mathrm{Y})$ and azimuth $(\Delta \mathrm{X})$ directions individually, and at $\Delta \mathrm{Y}$ and $\Delta \mathrm{X}$ directions simultaneously. With the tracking error at only $\Delta Y$, the focal spot moved upwards, from the lower rod to the upper rod (Fig 10a). With the tracking error at $\Delta X$, the focal spot moved along the laser rods (Fig 10b). With the simultaneous tracking error in $\Delta \mathrm{Y}$ and $\Delta \mathrm{X}$, the focal spot moved upwards to the upper rod and also to the right (Fig 10c).

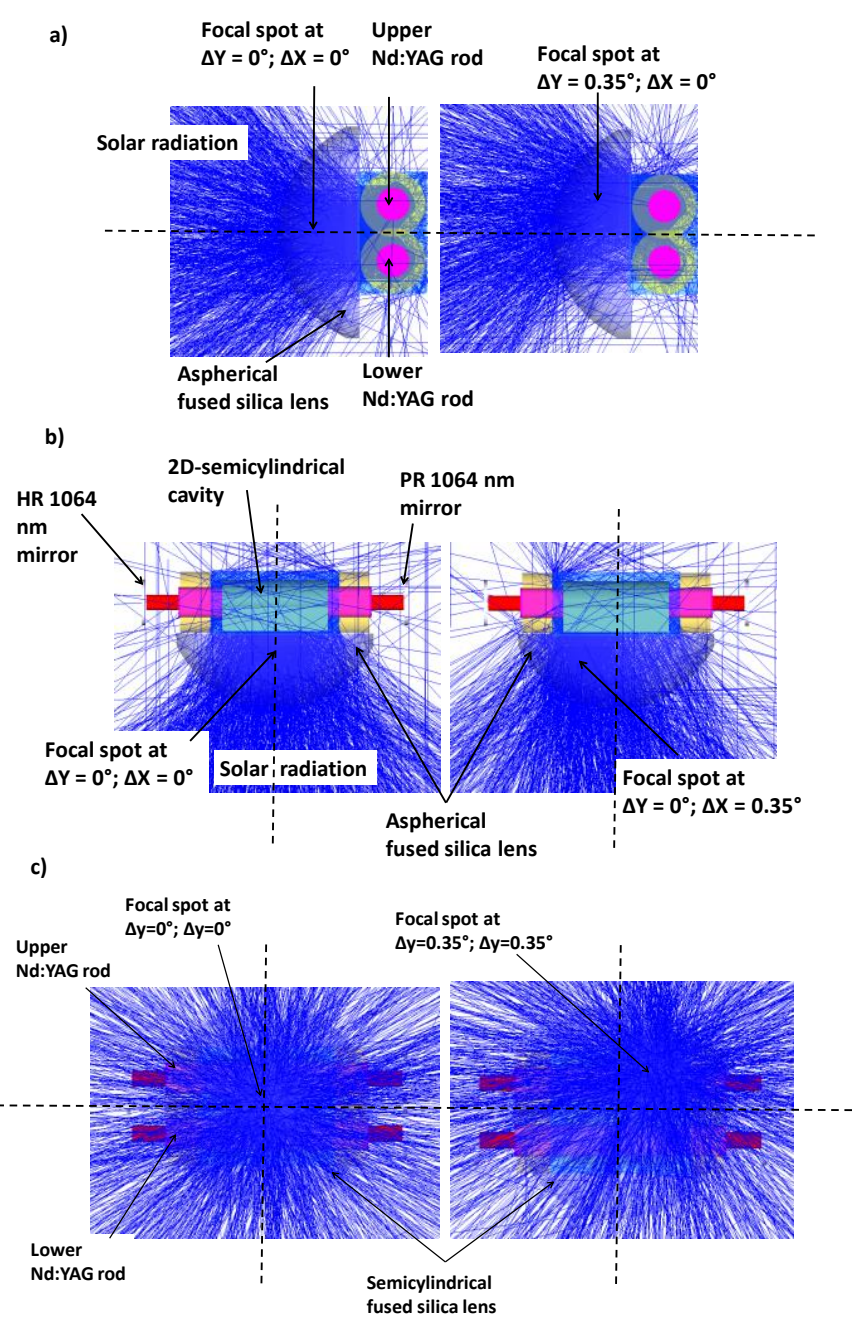

Fig. 10. Solar tracking error and its influence in the focal spot to pump the dual-rod scheme at a) altitude direction $(\Delta \mathrm{Y})$, $\mathrm{b})$ azimuth direction $(\Delta \mathrm{X})$ and c) $\Delta \mathrm{Y}$ and $\Delta \mathrm{X}$ simultaneously. 
In Fig. 11 is shown the absorbed pump flux distribution along the longitudinal cross-section of the dual-rod and the single-rod pumped with solar tracking error at $\Delta \mathrm{Y}$ and $\Delta \mathrm{X}$. The laser output powers are also indicated.
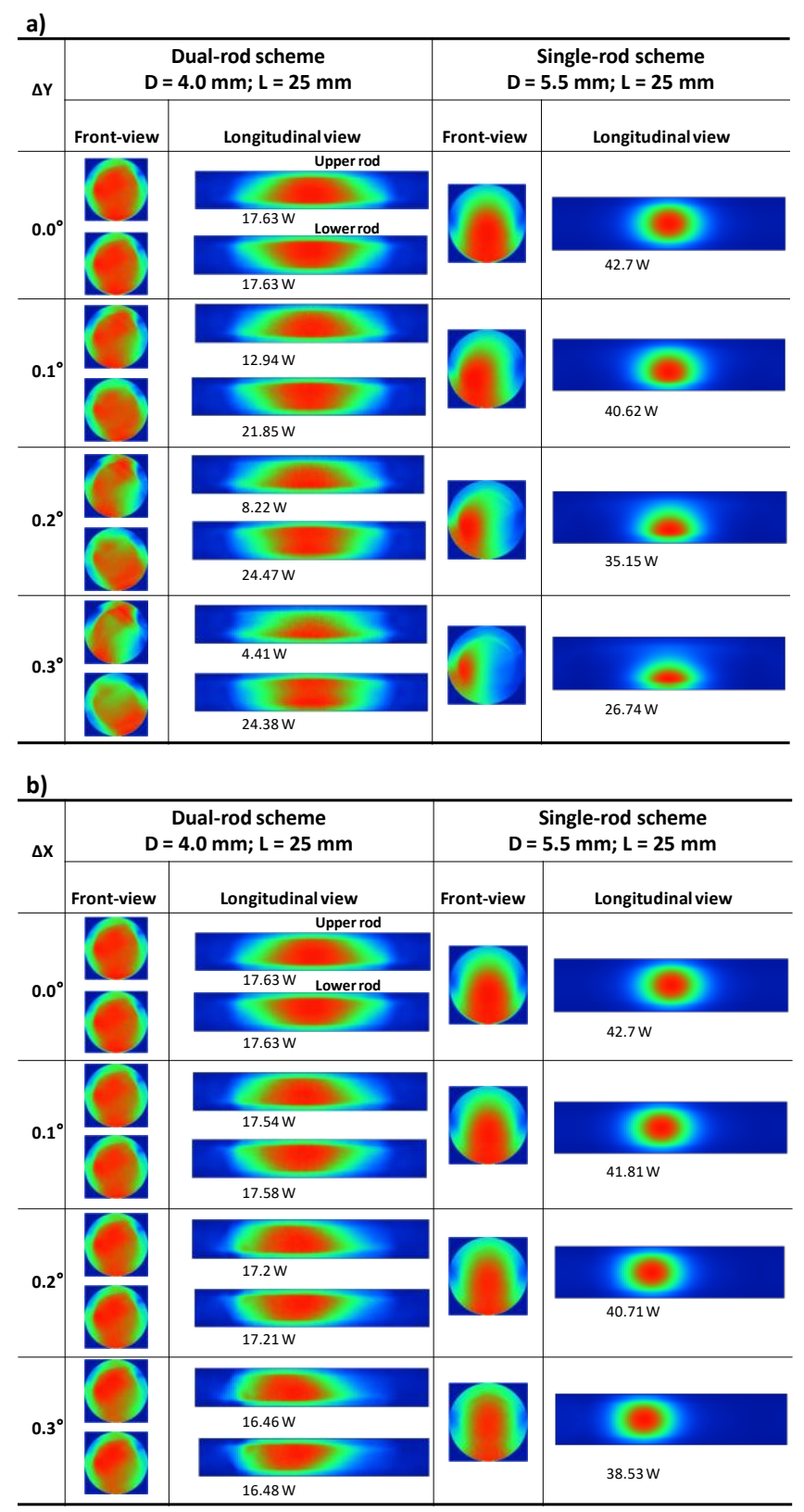

Fig. 11. Absorbed pump flux distribution along the longitudinal cross-section of the Nd:YAG crystal rods, for the dual-rod and the single-rod schemes, pumped with solar tracking error at a) $\Delta \mathrm{X}$ and b) $\Delta \mathrm{Y}$. The laser output powers numerically calculated are also shown.

With the tracking error at $\Delta \mathrm{Y}$, the pump flux distribution shifted upwards onto the upper rod of the dual-rod scheme, increasing its laser power, while that of the lower rod decreased. With the tracking error at $\Delta \mathrm{X}$, the absorbed pump flux distribution displaced along the laser rods.

For both the single and the dual-rod schemes, the normalized total multimode laser output power as a function of the $\Delta \mathrm{Y}$ and $\Delta \mathrm{X}$ solar tracking errors ranging from $0.00^{\circ}$ to $0.35^{\circ}$, is shown in Fig. 12. Normalized laser power for each laser rod (both upper and lower rod)of the dual-rod scheme is also given. For 
comparison, the normalized total laser output power of the previous dual-rod scheme is also provided [24]. The present dual-rod scheme using an aspheric lens and a compact pump cavity resulted in $34.2 \%$ more laser output power, $34.3 \%$ more collection efficiency, $61.1 \%$ more brightness figure of merit, $38 \%$ and $66.1 \%$ more tracking error compensation capacity in $\Delta \mathrm{Y}$ and $\Delta \mathrm{X}$ errors, respectively, in comparison to the previous dual-rod scheme with a semicylindrical lens and a less efficient pump cavity [24]. The laser rods were also placed much closer to the output end face of the fused silica aspheric lens, as compared to that of the previous scheme. Large diameter laser rods significantly improved both solar laser efficiency and tracking error compensation capacity, as shown in Fig. 12.

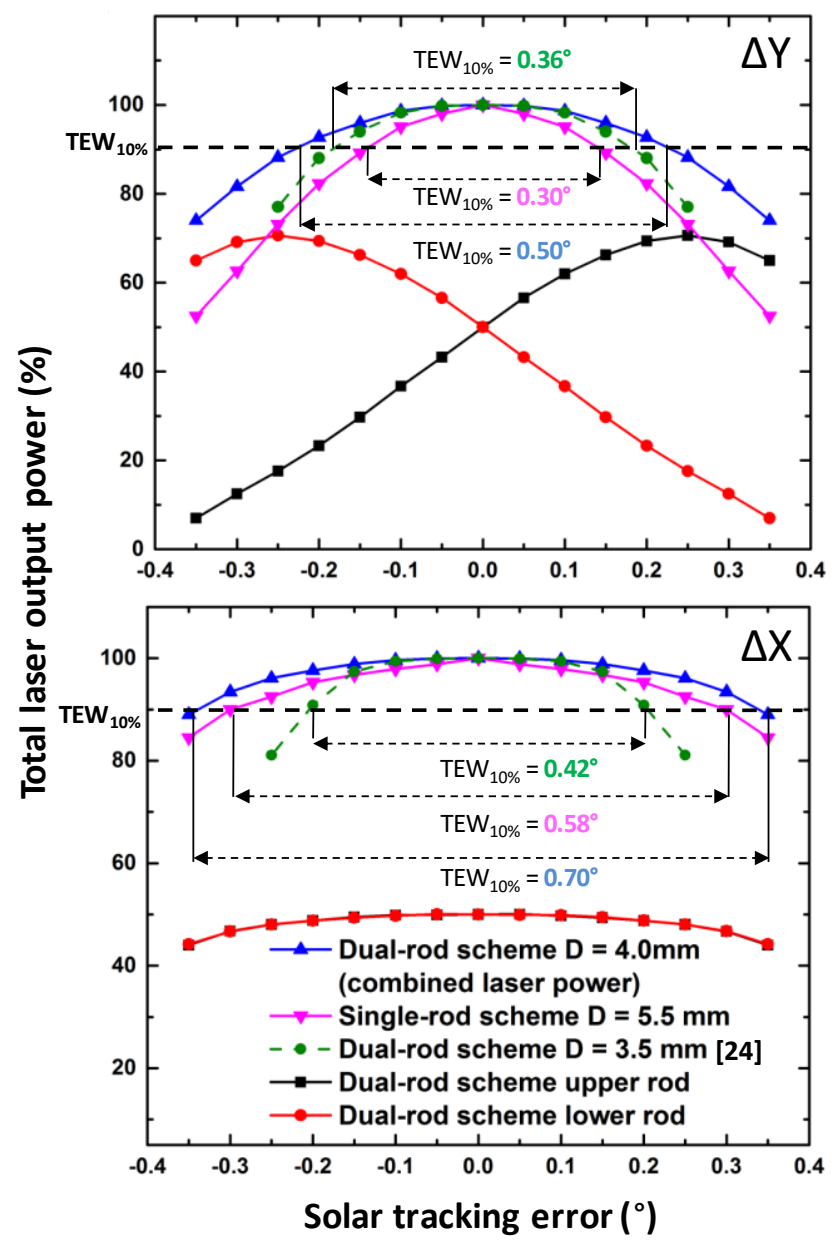

Fig. 12. Normalized total multimode laser output power, from the optimal alignment $\left(0.0^{\circ}\right)$ of the solar energy collection and concentration system, as a function of the $\Delta \mathrm{Y}$ and $\Delta \mathrm{X}$ solar tracking errors, for the single and the dual-rod schemes and also for the previous dual-rod scheme [24]. It is also shown the normalized power for each laser rod from the dual-rod scheme (both upper and lower rod), as well the tracking error width at $10 \%$ laser power loss $\left(\mathrm{TEW}_{10 \%}\right)$.

It is also represented the tracking error width at $10 \%$ laser power loss. The total combined laser power from both rods remained more stable than that of the single-rod scheme, due to the enlarged tracking error compensation capacity using two rods in a same pump cavity. The TEW $10 \%$ of the dual-rod scheme with $\Delta \mathrm{Y}$ error improved 1.66 times in relation to the single-rod scheme with $5.5 \mathrm{~mm}$ diameter rod. The $\mathrm{TEW}_{10 \%}$ of the dual-rod scheme with $\Delta \mathrm{X}$ error improved 1.21 times in relation to the single-rod scheme.

The absorbed pump flux distribution along the longitudinal cross-section of both the single and the dual Nd:YAG laser rods, pumped with simultaneous $\Delta \mathrm{Y}$ and $\Delta \mathrm{X}$ solar tracking errors, is presented in Fig. 13, along with the numerically obtained laser output powers. 


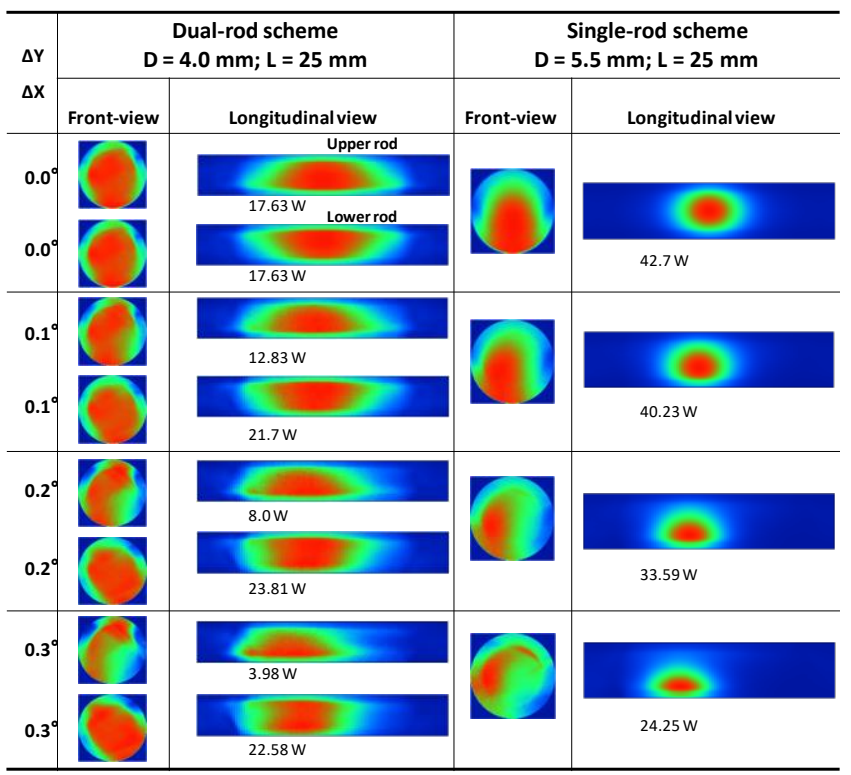

Fig. 13. Absorbed pump flux distribution along the longitudinal cross-section of both the single and the dual Nd:YAG crystal rods, pumped with solar tracking error at $\Delta \mathrm{Y}$ and $\Delta \mathrm{X}$ simultaneously. The laser output powers numerically calculated are also shown.

The dual-rod scheme presented a large improvement in tracking error compensation capacity comparatively to that of the single-rod scheme in both $\Delta \mathrm{X}$ and $\Delta \mathrm{Y}$ variations of the pump flux.

The numerical comparative results obtained are summarized in table 4. It is also shown the previous dual-rod scheme with tracking error capacity [24].

Table 4. Numerically calculated tracking error width at $10 \%$ laser power loss, for the singlerod and the dual-rod schemes and the previous dual-rod scheme [24]. The numerically calculated improvement in tracking error capacity using the dual-rod pumping scheme of the present work is also indicated.

\begin{tabular}{|c|c|c|c|c|}
\hline & \multicolumn{2}{|c|}{$\begin{array}{l}\text { Tracking error width at } 10 \% \\
\text { laser power loss }\end{array}$} & \multicolumn{2}{|c|}{$\begin{array}{l}\text { Improvement of the dual-rod } \\
\text { scheme of the present work } \\
\text { in relation to the single-rod } \\
\text { scheme and [24] (times) }\end{array}$} \\
\hline Schemes & $\Delta Y$ & $\Delta X$ & $\Delta \mathrm{Y}$ & $\Delta \mathrm{X}$ \\
\hline $\begin{array}{c}\text { Dual-rod } \\
\begin{array}{c}(\mathrm{D}=4.0 \mathrm{~mm} ; \\
\mathrm{L}=25)\end{array}\end{array}$ & 0.50 & 0.70 & - & - \\
\hline $\begin{array}{l}\begin{array}{c}\text { Single-rod } \\
(D=5.5 \mathrm{~mm} ; \\
L=25)\end{array}\end{array}$ & 0.30 & 0.58 & 1.66 & 1.21 \\
\hline $\begin{array}{c}\text { Dual-rod } \\
(3.5 \mathrm{~mm} ; \mathrm{L}=25)[24]\end{array}$ & 0.36 & 0.42 & 1.38 & 1.66 \\
\hline
\end{tabular}




\section{Discussion}

The dual-rod approach can be an alternative concept to achieve improved multimode solar laser output power with better beam quality and with improved tracking error compensation capacity. The significant alleviation in the thermal conditions of the laser medium helps to increase the laser performance [25].

The most efficient side-pumped solar laser experiment [18] used a $3.0 \mathrm{~mm}$ diameter and $30 \mathrm{~mm}$ length Nd:YAG rod. A fused silica aspherical lens efficiently focused the concentrated solar power from the focal zone of the parabolic mirror into the laser rod within a two-dimensional semicylindrical pump cavity. For $770 \mathrm{~W} / \mathrm{m}^{2}$ solar irradiance and $0.9 \mathrm{~m}^{2}$ effective collection area, $15.80 \mathrm{~W}$ multimode solar laser power was experimentally obtained. The LASCAD ${ }^{\odot}$ numerical analysis method, mentioned in Section 3.2, was then used to analyze the multimode solar laser output power of the single-rod scheme presented in this work, with the same conditions of effective area, solar irradiance and resonant cavity length [18]. For a $3.0 \mathrm{~mm}$ diameter and $30 \mathrm{~mm}$ length rod, $15.40 \mathrm{~W}$ laser output power was numerically obtained with the single-rod scheme presented in this work, revealing its accuracy of our numerical analysis in determining the multimode laser output power. More importantly, it was also numerically evaluated the previous solar laser scheme [18] with the same solar irradiance, effective collection area, rod dimensions and resonant cavity length as used in the present work. Only 35.0 W laser output power was numerically obtained, revealing the superiority of the present scheme. The LASCAD ${ }^{\odot}$ software presented a good agreement in the calculated results, in relation to the measured output laser power performance, as shown in Table 5.

Table 5. Comparison between the $\operatorname{LASCAD}^{\odot}$ numerical results of the single-rod scheme of this work with the most efficient side-pumped solar laser experiment[18].

\begin{tabular}{c|cc|cc}
\hline Parameter & $\begin{array}{c}\text { Single-rod } \\
\text { scheme from } \\
\text { this work }\end{array}$ & $\begin{array}{c}\text { Experimental results } \\
\text { from [18] }\end{array}$ & $\begin{array}{c}\text { Single-rod } \\
\text { scheme from } \\
\text { this work }\end{array}$ & $\begin{array}{c}\text { Numerical } \\
\text { results of [18] }\end{array}$ \\
\hline $\begin{array}{c}\text { Diameter } \\
\text { Length (mm) }\end{array}$ & 3.0 & 3.0 & 5.5 & 5.5 \\
$\begin{array}{c}\text { Solar irradiance } \\
\left(\text { W/m }{ }^{2}\right)\end{array}$ & 70 & 30 & 25 & 25 \\
$\begin{array}{c}\text { Effective } \\
\text { collection area } \\
\left(\mathrm{m}^{2}\right)\end{array}$ & 0.9 & 770 & 890 & \\
$\begin{array}{c}\text { Multimode laser } \\
\text { power (W) }\end{array}$ & 15.40 & 15.80 & 42.56 & \\
\hline
\end{tabular}

Other laser materials can also be pumped by these concepts, such as alexandrite crystal media [26], composite laser rods such as YAG-Nd:YAG-YAG undoped-doped-undoped, and Nd:YAG grooved rods [17], Cr:Nd:YAG ceramic medium [11] and Cr: LiCAF [27]. New schemes of solar-pumped lasers, such as luminescent solar concentrator [28] and diffusive scattering cooling liquid [29]can also be further improved in their performances.

\section{Conclusions}

Two side-pumped Nd:YAG solar laser configurations were studied in this work, the single-rod and the dual-rod pumping schemes. The former pumped a thick laser rod with the full collection area and the latter pumped two thin laser rods simultaneously, each one using half of the collection area. Both the configurations were composed of the fused silica aspheric lens and the 2D-shaped-semicylindrical pump cavity, within which the Nd:YAG rods were mounted, allowing a tight focusing of the concentrated solar pump power from the focal spot of the heliostat- 
parabolic mirror solar energy collection and concentration system and an efficient pumping of the laser crystals. The multimode solar laser output power, the threshold pump power, and the slope efficiency of the single-rod, the dualrod with two laser beams, and the dual rod with one laser beam schemes were calculated both numerically and analytically. The optimized optical pumping system were found through ZEMAX ${ }^{\odot}$ software while the optimum solar laser power and beam parameters were found using $\operatorname{LASCAD}^{\odot}$ numerical analysis. The obtained results are shown in Table 6.

Table 6. Numerically calculated laser output power, collection efficiency and solar-to-laser power conversion efficiency for the single-rod and the dual-rod schemes, and its comparison with the most efficient experimental side-pumped solar laser of Liang et al [18]. It is also shown the collection areas and the solar irradiance.

\begin{tabular}{c|ccccc}
\hline & & \multicolumn{2}{c}{ Present numerical work } & \multicolumn{2}{c}{ Improvements (times) } \\
\cline { 2 - 6 } Parameters & {$[18]$} & $\begin{array}{c}\text { Single-rod } \\
\text { scheme }\end{array}$ & $\begin{array}{c}\text { Dual-rod } \\
\text { scheme }\end{array}$ & $\begin{array}{c}\text { Single-rod } \\
\text { scheme to } \\
{[18]}\end{array}$ & $\begin{array}{c}\text { Dual-rod } \\
\text { scheme to } \\
{[18]}\end{array}$ \\
\hline $\begin{array}{c}\text { Collection area } \\
\left(\mathrm{m}^{2}\right)\end{array}$ & 0.9 & 1.56 & 1.56 & - & - \\
$\begin{array}{c}\text { Solar irradiance } \\
\left(\mathrm{W} / \mathrm{m}^{2}\right)\end{array}$ & 770 & 890 & 890 & - & - \\
$\begin{array}{c}\text { Laser output } \\
\text { power (W) }\end{array}$ & 15.8 & 42.70 & 37.72 & - & 1.37 \\
$\begin{array}{c}\text { Collection } \\
\left.\text { efficiency (W/m }{ }^{2}\right)\end{array}$ & 17.6 & 27.37 & 24.18 & 1.55 & 1.19 \\
$\begin{array}{c}\text { Solar-to-laser } \\
\text { power conversion } \\
\text { efficiency (\%) }\end{array}$ & 2.43 & 3.26 & 2.88 & 1.34 & \\
\hline
\end{tabular}

The single-rod scheme obtained $42.70 \mathrm{~W}$ solar laser power, corresponding to $27.37 \mathrm{~W} / \mathrm{m}^{2}$ collection efficiency and 3.26\% solar-to-laser power conversion efficiency, being 1.55 and 1.34 times, respectively, more than the previous experimental records in side-pumping solar laser configurations [18]. The dual-rod scheme achieved a lower value, $37.72 \mathrm{~W}$ solar laser power, but still corresponding to $24.20 \mathrm{~W} / \mathrm{m}^{2}$ collection efficiency and $2.88 \%$ solar-to-laser power conversion efficiency, being 1.34 and 1.19 times, respectively, more than the previous records in side-pumping configurations [18]. On the other hand, the dual-rod with two laser beams and one laser beam schemes presented a largely reduced $\mathrm{M}^{2}$ factors, obtaining 4.8 and 5.8 times more brightness figure of merit, respectively, than that of the single-rod scheme.

The thermal performance of the dual-rod configurations also showed a large enhancement in relation to the singlerod scheme, achieving a decrease in heat load, temperature and stress intensity of about $64.2 \%, 77 \%$ and $79 \%$, respectively. A large improvement in tracking error compensation capacity was numerically achieved with the dualrod pumping approach, where the $\mathrm{TEW}_{10 \%}$ was improved 1.66 and 1.21 times in altitude $(\Delta \mathrm{Y})$ and azimuth $(\Delta \mathrm{X})$ errors, respectively, as compared to the numerically simulated side-pumped single-rod scheme with a thick rod.

Funding Information Financial support of the strategic project (UID/FIS/00068/2019) of the Science and Technology Foundation of Portuguese Ministry of Science, Technology and Higher Education (FCT - MCTES) is acknowledged.

Acknowledgment The fellowship grants PD/BD/128267/2016, PD/BD/142827/2018, SFRH/BPD/125116/2016 and CEECIND/03081/2017 of B. D. Tibúrcio, D. Garcia, C. R. Vistas and J. Almeida, respectively, are acknowledged. 


\section{References}

[1] M. Lando, J.A. Kagan, Y. Shimony, Y.Y. Kalisky, Y. Noter, A. Yogev, S.R. Rotman, S. Rosenwaks, Solar-pumped solid state laser program, SPIE1997.

[2] Y. Takashi, O. Tomomasa, D.T. Hung, K. Hiroki, N. Junichi, O. Kouta, Demonstration of Solar-Pumped Laser-Induced Magnesium Production form Magnesium Oxide, in: S.N. Mathaudhu, W.H. Sillekens, N.R. Neelameggham, N. Hort (Eds.) Magnesium Technology 2012, Springer International Publishing, Cham, 2016, pp. 55-58.

[3] T. Yabe, S. Uchida, K. Ikuta, K. Yoshida, C. Baasandash, M.S. Mohamed, Y. Sakurai, Y. Ogata, M. Tuji, Y. Mori, Y. Satoh, T. Ohkubo, M. Murahara, A. Ikesue, M. Nakatsuka, T. Saiki, S. Motokoshi, C. Yamanaka, Demonstrated fossil-fuel-free energy cycle using magnesium and laser, Applied Physics Letters, 89 (2006) 261107.

[4] C.G. Young, A Sun-Pumped cw One-Watt Laser, Appl. Opt., 5 (1966) 993-997.

[5] M. Weksler, J. Shwartz, Solar-pumped solid-state lasers, IEEE Journal of Quantum Electronics, 24 (1988) 1222-1228.

[6] H. Arashi, Y. Oka, N. Sasahara, A. Kaimai, M. Ishigame, A Solar-Pumped cw 18 W Nd:YAG Laser, Japanese Journal of Applied Physics, 23 (1984) 1051-1053.

[7] R.M.J. Benmair, J. Kagan, Y. Kalisky, Y. Noter, M. Oron, Y. Shimony, A. Yogev, Solar-pumped Er,Tm,Ho:YAG laser, Optics Letters, 15 (1990) $36-$ 38.

[8] M. Lando, J. Kagan, B. Linyekin, V. Dobrusin, A solar-pumped Nd:YAG laser in the high collection efficiency regime, Optics Communications, 222 (2003) 371-381.

[9] D. Liang, J. Almeida, Highly efficient solar-pumped Nd:YAG laser, Optics Express, 19 (2011) 26399-26405.

[10] T.H. Dinh, T. Ohkubo, T. Yabe, H. Kuboyama, 120 watt continuous wave solar-pumped laser with a liquid light-guide lens and an Nd:YAG rod, Optics Letters, 37 (2012) 2670-2672.

[11] T. Yabe, T. Ohkubo, S. Uchida, K. Yoshida, M. Nakatsuka, T. Funatsu, A. Mabuti, A. Oyama, K. Nakagawa, T. Oishi, K. Daito, B. Behgol, Y. Nakayama, M. Yoshida, S. Motokoshi, Y. Sato, C. Baasandash, High-efficiency and economical solar-energy-pumped laser with Fresnel lens and chromium codoped laser medium, 90 (2007) 261120

[12] P. Xu, S. Yang, C. Zhao, Z. Guan, H. Wang, Y. Zhang, H. Zhang, T. He, High-efficiency solar-pumped laser with a grooved Nd:YAG rod, Appl. Opt., 53 (2014) 3941-3944.

[13] L. Jing, H. Liu, Y. Wang, W. Xu, H. Zhang, Z. Lu, Design and Optimization of Fresnel Lens for High Concentration Photovoltaic System \%J International Journal of Photoenergy, 2014 (2014) 7.

[14] D. Liang, J. Almeida, C.R. Vistas, $25 \mathrm{~W} / \mathrm{m}^{2}$ collection efficiency solar-pumped Nd:YAG laser by a heliostat-parabolic mirror system, Appl. Opt., 55 (2016) 7712-7717.

[15] D. Liang, J. Almeida, C.R. Vistas, E. Guillot, Solar-pumped Nd:YAG laser with $31.5 \mathrm{~W} / \mathrm{m}^{2}$ multimode and $7.9 \mathrm{~W} / \mathrm{m}^{2} \mathrm{TEM}$ oo-mode collection efficiencies, Solar Energy Materials and Solar Cells, 159 (2017) 435-439.

[16] D. Liang, C.R. Vistas, B.D. Tiburcio, J. Almeida, Solar-pumped Cr:Nd:YAG ceramic laser with 6.7\% slope efficiency, Solar Energy Materials and Solar Cells, 185 (2018) 75-79.

[17] C. Zhao, Z. Guan, H. Zhang, How to harvest efficient laser from solar light, SPIE2018.

[18] D. Liang, C.R. Vistas, J. Almeida, B.D. Tibúrcio, D. Garcia, Side-pumped continuous-wave Nd:YAG solar laser with $5.4 \%$ slope efficiency, Solar Energy Materials and Solar Cells, 192 (2019) 147-153.

[19] D. Liang, J. Almeida, Solar-Pumped TEMoo-Mode Nd:YAG laser, Optics Express, 21 (2013) 25107-25112.

[20] Standard Tables for Reference Solar Spectral Irradiances: Direct Normal and Hemispherical on $37^{\circ}$ Tilted Surface, ASTM G173-03 International, West Conshohocken, PA, 2012.

[21] C.Z. B. Zhao, J. He, and S. Yang, The study of active medium for solar-pumped solid-state lasers, Acta Opt. Sin., 27 (2007) 1797 - 1801

[22] W. Koechner, Solid-State Laser Engineering, 6 ed., Springer-Verlag, New York, 2006.

[23] M. Schelhorn, High Power diode-pumped Tm:YLF Laser, Appl. Phy. B, Laser and Optics, 91 (2008) 71-74

[24] B.D. Tibúrcio, D. Liang, J. Almeida, D. Garcia, C.R. Vistas, Dual-rod pumping approach for tracking error compensation in solar-pumped lasers, Journal of Photonics for Energy, 9 (2019).

[25] W. Koechner, Thermal Lensing in a Nd:YAG Laser Rod, Appl. Opt., 9 (1970) 2548-2553.

[26] M. Lando, Y. Shimony, R.M.J. Benmair, D. Abramovich, V. Krupkin, A. Yogev, Visible solar-pumped lasers, Optical Materials, 13 (1999) $111-$ 115.

[27] S. Payziyev, K. Makhmudov, Solar pumped Nd:YAG laser efficiency enhancement using Cr:LiCAF frequency down-shifter, Optics Communications, 380 (2016) 57-60.

[28] P.D. Reusswig, S. Nechayev, J.M. Scherer, G.W. Hwang, M.G. Bawendi, M.A. Baldo, C. Rotschild, A path to practical Solar Pumped Lasers via Radiative Energy Transfer, Scientific Reports, 5 (2015) 14758.

[29] C.J.C. Smyth, S. Mirkhanov, A.H. Quarterman, K.G. Wilcox, $27.5 \mathrm{~W} / \mathrm{m}^{2}$ collection efficiency solar laser using a diffuse scattering cooling liquid, Appl. Opt., 57 (2018) 4008-4012. 